\title{
A pesca comercial das sardinhas (Triportheus spp.) desembarcadas no mercado pesqueiro de Porto Velho, Rondônia (1990-2004): Produção pesqueira e perfil geral
}

\author{
Carolina Rodrigues da Costa Doria* \\ Luiz Jardim de Queiroz \\ Departamento de Biologia, Laboratório de Ictiologia e Pesca \\ Universidade Federal de Rondônia (UNIR), BR 364, km 9,5, sentido Rio Branco, Porto Velho. CEP 78900-000, Porto Velho - RO, Brasil. \\ Autor para correspondência: \\ carolinarcdoria@uol.com.br
}

Submetido em 20/07/2007

Aceito para publicação em 06/05/2008

\section{Resumo}

O perfil da pesca das sardinhas, gênero Triportheus, desembarcadas no mercado pesqueiro do flutuante Cai N'água no município de Porto Velho (RO) entre 1990 e 2004, é analisado verificando variações inter e intra-anuais na produção e nos comprimentos, bem como características gerais da pesca. A produção anual variou de 15.372 a $78.340 \mathrm{~kg}$ ( édia $=33.482 \mathrm{~kg}$ ), totalizando $5,46 \%$ da produção total no período estudado. Essas variações podem estar relacionadas às mudanças no esforço pesqueiro, como reflexo da busca por alternativas de pescado, ou ainda ao nível hidrológico, já que produções anuais mais altas foram precedidas pelos anos com períodos de cheias mais intensas. A produção mensal variou de 0 a $21.456 \mathrm{~kg}$ (média $=2.876 \mathrm{~kg}$ ). Maiores valores da produção mensal foram obtidos em períodos de menor nível hidrológico ( $\operatorname{seca}$ ) $[\mathrm{r}$ (Pearson) $=-0,53$; $\mathrm{p}<0,0001]$. As capturas médias por pescador/dia entre 2003 e 2004 foram de 9,27kg. Rios foram os principais ambientes de captura, principalmente os rios Jamari, Machado e Novo Aripuanã, afluentes do rio Madeira. Os comprimentos variaram de 70 a 380mm e apresentaram padrão bimodal, sugerindo a presença de mais de uma espécie nas capturas.

Unitermos: pesca, esforço pesqueiro, Rio Madeira, Amazônia

\section{Abstract}

The commercial fishing of sardines (Triportheus spp.) landed in the fishery market of Porto Velho, Rondônia (1990-2004): Yield and general profile. The profile of sardine fishing, the Triportheus species, landed at the fishing market of Porto Velho (RO) between 1990 and 2004, was established by analyzing variations in the intra- and inter-annual yield as well as the length and general characteristics of the fishing. The annual production varied from 15,372 to $78,340 \mathrm{~kg}$ (average $=33,482 \mathrm{~kg}$ ), totaling $5.46 \%$ of the overall production during the studied period. These variations can be related to the changes in the fishermen's effort, as a reflex to searching for alternatives for fishing, or related to the level of the river, since the greatest annual yields were preceded by years of having more intense flooding. The monthly production varied from 0 to $21,456 \mathrm{~kg}$ (average $=2,876 \mathrm{~kg}$ ). Greater values of production were obtained in periods of lower hydrological levels (dry season) $[\mathrm{r}($ Pearson $)=-0.53 . \mathrm{p}<0.0001]$. The average capture per fisherman/day between 2003 and 2004 was $9.27 \mathrm{~kg}$. Rivers were the main environment from which the captures took place, mainly from the Jamari, Ma- 
chado and Novo Aripuanã rivers, that are tributaries of the Madeira River. The lengths of the fishes varied from 70 to $380 \mathrm{~mm}$ and presented a bimodal pattern, suggesting the presence of more than one species within the captures.

Key words: fishing, effort, Madeira River, Amazônia

\section{Introdução}

A atividade pesqueira sempre desempenhou papel de destaque no contexto econômico e social da Amazônia, constituindo-se numa das mais valiosas e tradicionais atividades extrativistas da região (Petrere Jr., 1982; Santos, 1986/87; Santos e Ferreira, 1999). O próprio processo de ocupação da Amazônia, que se desencadeou após os séculos XVII e XVIII e que foi centralizado ao longo das calhas dos principais rios da região, é uma representação da importância dos rios e seus respectivos recursos naturais na vida do homem amazônico (Santos e Santos, 2005).

A pesca na região amazônica destaca-se pela alta produtividade, pela dependência da população tradicional por este recurso, cujo consumo per capita supera $600 \mathrm{~g} / \mathrm{dia}$, e principalmente pela imensa riqueza de espécies exploradas (Barthem e Fabré, 2004).

Apesar de muitas espécies serem capturadas na pesca comercial na Amazônia, o crescente esforço de pesca sobre determinados grupos de espécies tem provocado a redução nos rendimentos de muitos estoques (Petrere Jr., 1978; Santos, 1986/87; Boischio, 1992). Devido a isto, o interesse comercial sobre espécies de menor porte e valor comercial tem aumentado substancialmente em diversas partes da Amazônia, como um reflexo da busca por novas alternativas de pescado (Tello e Bayley, 2001; Batista e Petrere Jr., 2003).

Nesse contexto, as espécies do gênero Triportheus Cope, 1872, conhecidas na região por sardinhas, se constituem num importante recurso utilizado na alimentação de comunidades tradicionais (Cerdeira et al., 1997; Batista et al., 1998; Amaral, 2005). Nos últimos anos, essa categoria tem sido um dos principais alvos da pesca comercial em praticamente toda a Amazônia brasileira (Santos, 1986/87; Batista, 1998; Batista e Petrere Jr., 2003; Barthem, 2004; Viana, 2004). Barthem e Fabré (2004), realizando uma revisão de informações de desembarques pesqueiros ao longo da década de 90 de diversos locais ao longo do rio Solimões/Amazonas, verificaram que as sardinhas representaram 2,5\% do desembarque total dos principais portos, desde Iquitos até Belém. Apenas para a cidade de Manaus, entre 1994 e 1996, as sardinhas estiveram entre as seis espécies mais comercializadas, representando mais de $4 \%$ de toda a produção na cidade (Batista, 2004).

Nos desembarques pesqueiros do Estado de Rondônia, tem sido registrada a presença de três espécies de sardinhas: Triportheus albus, T. angulatus e T. auritus (Santos, 1986/87) sob os nomes de sardinha comum, sardinha papuda e sardinha comprida, respectivamente (Ferreira et al., 1998). Embora os pescadores reconheçam as diferentes espécies no desembarque, são registradas em conjunto em relatórios de produção pesqueira como "sardinhas".

Assim, o objetivo deste trabalho foi analisar o perfil da pesca da categoria sardinha, representada pelas espécies do gênero Triportheus, desembarcada no mercado pesqueiro de Porto Velho (RO) entre 1990 e 2004. A proposta foi analisar variações inter e intra-anuais na produção e variações inter-anuais nos comprimentos desembarcados. Além disso, é apresentada uma caracterização geral dos principais aspectos relacionados à pesca das sardinhas que podem contribuir ao manejo deste recurso multiespecífico.

\section{Material e Métodos}

\section{Área de estudo}

O mercado pesqueiro Cai n'água da cidade de Porto Velho configura-se como o principal do Estado de Rondônia (Goulding, 1979) e da Amazônia Ocidental (Santos, 1986/87). Constitui-se num importante elo entre duas grandes áreas de produção pesqueira da região: uma situada na Amazônia Central e outra no vale dos rios Beni-Mamoré-Guaporé (Santos, 1986/87).

A produção do pescado em Porto Velho se situa em torno de 1000 toneladas por ano e a pesca que abastece esse mercado é realizada em sua maior parte nos cursos médio e 
inferior do Madeira, trecho onde as planícies de inundação são mais extensas e semelhantes às da Amazônia Central. As áreas de pescaria compreendem, principalmente, as regiões da foz dos rios Jamari, Machado, Aripuanã e Canumã e as cachoeiras localizadas a montante da cidade de Porto Velho (Santos, 1986/87; LEME, 2005).

\section{Obtenção e análise dos dados}

Os dados de produção das sardinhas em quilos (kg) anual e mensal referente aos anos de 1990 e 2004 foram obtidos de registros da Colônia de Pescadores de Porto Velho, Rondônia, Tenente Santana - Z1. A colônia de pescadores registra diariamente toda a produção desembarcada e comercializada no seu flutuante (Cai N'água) por pescadores filiados, sobre a qual é cobrada a taxa obrigatória de comercialização do pescado que é destinada à colônia. Estes dados são registrados, processados e armazenados por funcionários da administração da própria colônia.

A produção mensal foi correlacionada por meio da Correlação de Pearson ao nível hidrológico do rio Madeira (Zar, 1999). Este, por sua vez, foi obtido na Estação Portobrás, localizada em Porto Velho, a partir da Companhia de Pesquisas em Recursos Minerais (CPRM) da mesma cidade.

Entrevistas foram realizadas com pescadores no período compreendido entre outubro de 2003 e outubro de 2004, a fim de identificar os rendimentos (CPUE - Captura Por Unidade de Esforço: biomassa desembarcada por número de pescadores por dia) e as principais características da pesca das sardinhas (p.ex., ambientes e artes de pesca, períodos de safra e entressafra, etc.). A seleção dos pescadores para aplicação do questionário foi aleatória, totalizando 306 entrevistas. Destas, foram selecionadas apenas as que continham o registro de captura de sardinhas.

Diariamente, exceto finais de semanas e feriados, coletores treinados e munidos com uma trena registraram dados de comprimento padrão em milímetros de exemplares desembarcados nos anos de 1997 e 1999 a 2004. A amostragem considerou pelo menos 20 exemplares de tamanhos variados do total desembarcado no dia no flutuante Cai N'água e terminal pesqueiro, na cidade de Porto Velho.

\section{Resultados}

A categoria sardinha contribuiu com $5,46 \%$ da produção total desembarcada no mercado pesqueiro de Porto Velho no período de 1990 a 2004, com média anual de $33.482 \mathrm{~kg}$ (Tabela 1). A produção anual entre os anos de 1990 e 1994 esteve entre 15.000 e $22.000 \mathrm{~kg}$, representando de 2 a $6 \%$ da produção total desembarcada. A partir do ano de 1994 observa-se um incremento na produção e na participação das sardinhas, com valores que variaram entre 20.000 e $78.000 \mathrm{~kg}$ e participação relativa variando entre 6 e 10\%, com uma aparente quebra nestas proporções nos anos de 1997 e 2003.

$\mathrm{Na}$ figura 1, podem ser observados os valores anuais dos desembarques das sardinhas acompanhados pelo nível hidrológico do rio Madeira. Aparentemente, valores mais altos na produção anual (1998/1999 e 2001/2002) foram precedidos por cheias mais intensas (acima de $1.600 \mathrm{~cm}$ ) e secas menos pronunciadas.

O rendimento médio entre outubro de 2003 e outubro de 2004 foi de 9,27kg/pescador/dia. Das 306 entrevistas realizadas neste período, em 33 foi registrada a captura de sardinhas. Rios foram os principais ambientes de captura destas espécies e os mais explorados foram: Jamari, Machado e Novo Aripuanã. As artes de pesca mais utilizada pelos pescadores foram malhadeiras e redes de lances (Tabela 2).

A produção pesqueira mensal variou de zero a $21.456 \mathrm{~kg}$ (média: $2.876 \pm 323$ ). Os maiores valores foram observados quando o nível do rio Madeira encontrava-se nas suas menores cotas, principalmente nos meses entre agosto e outubro (Figura 2), como comprovado pela correlação de Pearson $(r=-0,53 ; p<0,0001)$.

Os comprimentos das sardinhas variaram de 70 a $380 \mathrm{~mm}$ com média de $209,2 \mathrm{~mm}( \pm 46,4)$. Observase para todos os anos, exceto 2004, uma tendência à bimodalidade na estrutura em comprimento, isto é, a presença de dois picos nos histogramas (Figura 3). Nos anos de 1997 e 2001 a 2003, a pesca estava recrutando apenas indivíduos entre 70 e $270 \mathrm{~mm}$, enquanto que no último ano (2004) os indivíduos recrutados estavam entre as maiores classes (150 e 370mm). 
TABELA 1: Produção anual da categoria sardinha (Triportheus spp.) em quilos e percentagens da produção total desembarcada no mercado pesqueiro Cai N'Água em Porto Velho, Rondônia, no período de 1990 a 2004.

\begin{tabular}{cccc}
\hline \multirow{2}{*}{ Ano } & \multicolumn{2}{c}{ Produção } & \% \\
\cline { 2 - 3 } & $\begin{array}{c}\text { Total do mercado } \\
\text { de Porto Velho }\end{array}$ & Triportheus spp. \\
\hline 1990 & 614.080 & 19.330 & 3,15 \\
1991 & 742.260 & 17.500 & 2,36 \\
1992 & 391.604 & 15.605 & 3,98 \\
1993 & 1.060 .939 & 21.398 & 2,02 \\
1994 & 377.238 & 22.026 & 5,84 \\
1995 & 490.588 & 51.957 & 10,59 \\
1996 & 483.582 & 36.391 & 7,53 \\
1997 & 997.636 & 15.372 & 1,54 \\
1998 & 566.804 & 43.568 & 7,69 \\
1999 & 528.110 & 47.860 & 9,06 \\
2000 & 437.090 & 32.100 & 7,34 \\
2001 & 768.440 & 78.340 & 10,19 \\
2002 & 677.440 & 48.130 & 7,10 \\
2003 & 575.350 & 21.900 & 3,81 \\
2004 & 491.581 & 30.710 & 6,25 \\
\hline Média & $\mathbf{6 1 3 . 5 1 6}$ & $\mathbf{3 3 . 4 8 2}$ & $\mathbf{5 , 4 6}$ \\
\hline & & &
\end{tabular}

TABELA 2: Resumo das principais características da pesca das sardinhas (Triportheus spp.) desembarcadas no mercado pesqueiro de Porto Velho, Rondônia.

\begin{tabular}{l|c}
\hline \multicolumn{2}{c}{ Características } \\
\hline Ambientes de pesca & Rios \\
\hline Áreas de pesca & Baixo rio Madeira \\
\hline Principais rios & $\begin{array}{c}\text { Jamari, Machado e Novo } \\
\text { Aripuanã }\end{array}$ \\
\hline Aparelhos de pesca & $\begin{array}{c}\text { Malhadeiras e Redes de } \\
\text { lance }\end{array}$ \\
\hline Período de safra & Setembro a novembro \\
\hline Período de entressafra & Dezembro a agosto \\
\hline Comprimentos & Média: 209,2mm $\pm 46,4$ \\
\cline { 2 - 2 } & Amplitude: $70-380 \mathrm{~mm}$ \\
\hline $\begin{array}{l}\text { CPUE } \\
\text { Número de viagens com } \\
\text { captura de sardinhas regis- }\end{array}$ & $9,27 \mathrm{~kg} /$ pescador/dia $\pm 10,24$ \\
\hline $\begin{array}{l}\text { Número total de dias dedi- } \\
\text { cados à pesca }\end{array}$ & 33 \\
\hline
\end{tabular}

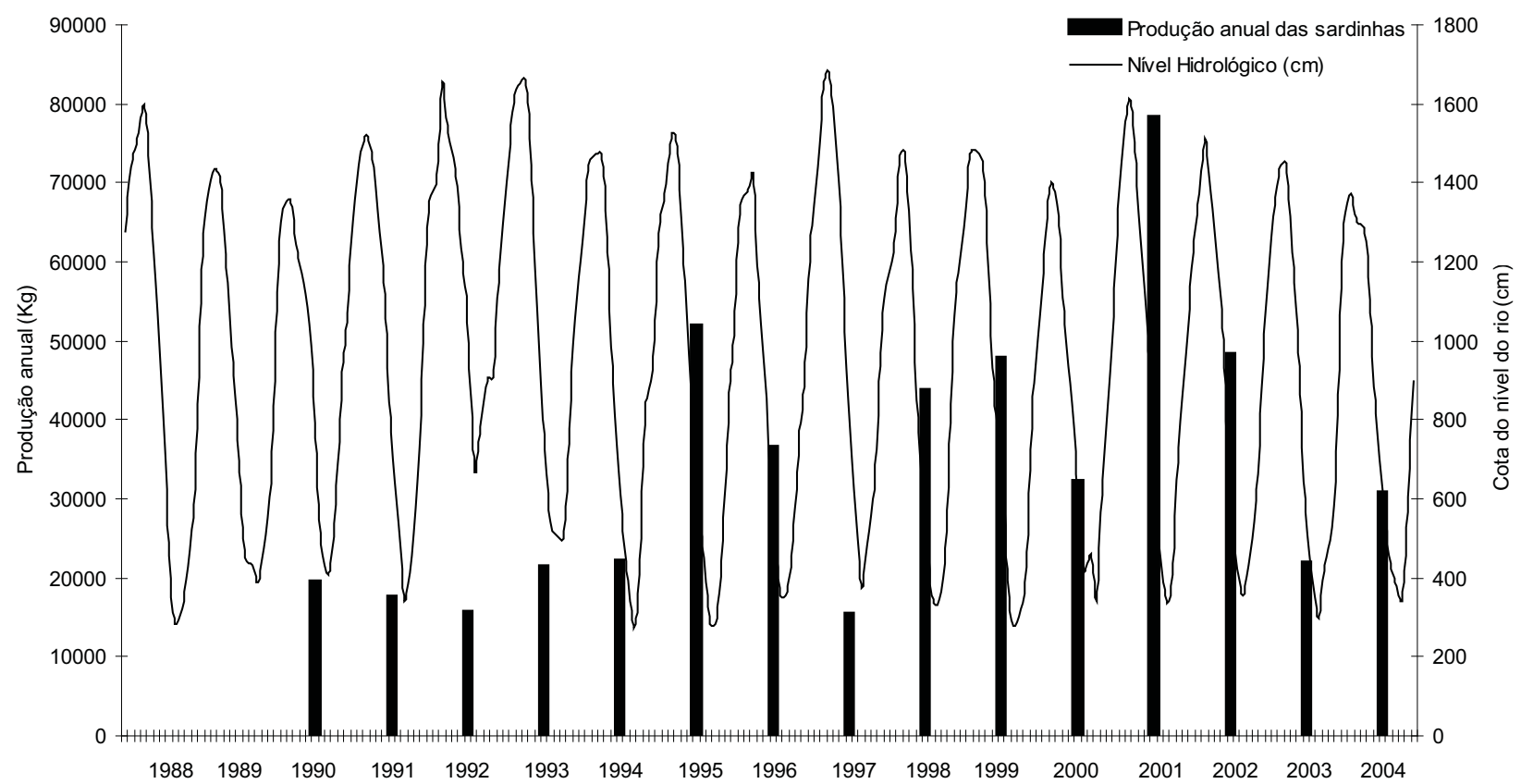

FIGURA 1: Produção anual da categoria sardinha (Triportheus spp.) em quilos (kg) desembarcada no mercado pesqueiro Cai N'Água em Porto Velho, Rondônia, no período de 1990 a 2004. (Fonte: Colônia de Pescadores Tentente Santana Z1 de Porto Velho). 


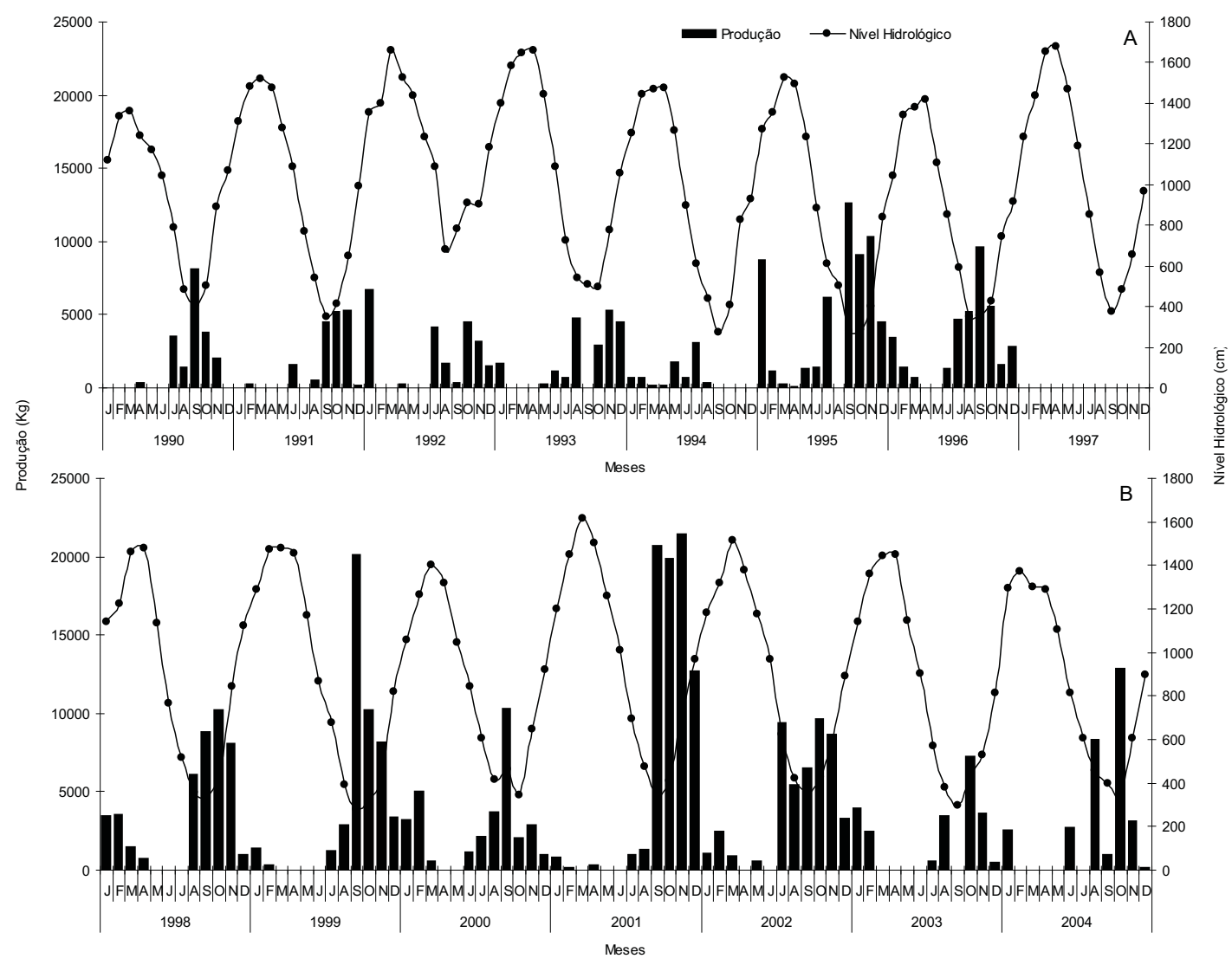

FIGURA 2: Produção mensal da categoria sardinha (Triportheus spp.) em quilos (kg) desembarcada no mercado pesqueiro Cai N'Água em Porto Velho, Rondônia, no período de 1990 a 2004.

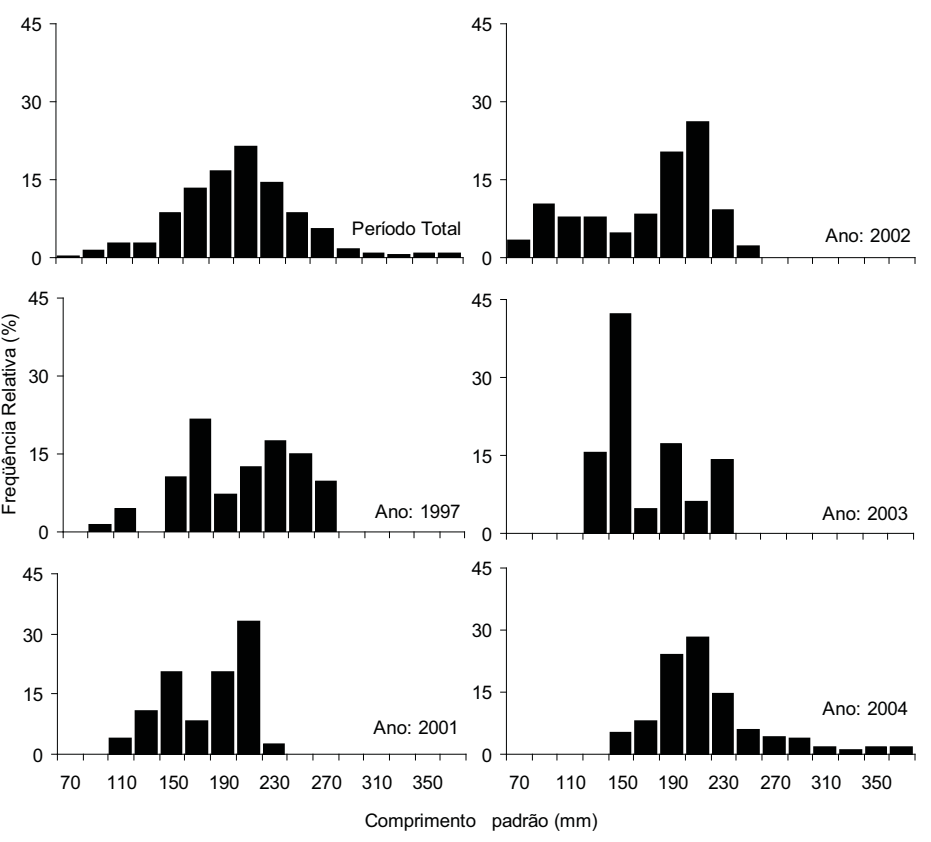

FIGURA 3: Composições de comprimento das sardinhas (Triportheus spp.) desembarcadas no mercado pesqueiro de Porto Velho, Rondônia, no período de 1997 e 2001 a 2004. 


\section{Discussão}

Goulding (1979) registrou uma produção de aproximadamente $15.000 \mathrm{~kg}$ de sardinhas no ano de 1977. Boischio (1992), por sua vez, avaliando a produção pesqueira em Porto Velho entre 1984 e 1989 das espécies mais relevantes, colocou as sardinhas na categoria "diversos", deixando implícito que sua produção também esteve próxima de $15.000 \mathrm{~kg}$ anuais. As estatísticas mais recentes (Figura 1) mostram que a partir de 1995 ocorrem valores mais altos e oscilações pronunciadas na produção das sardinhas. As oscilações que podem estar associadas a diversos fatores, entre eles a aumentos do esforço de pesca sobre esse estoque. Contudo, a ausência de informações históricas do esforço de pesca para todo o período estudado não nos permite abordar maiores considerações.

O aparente aumento da produção das sardinhas pode ser um reflexo da busca por novas fontes de pescado, como tem sido comum em várias regiões da Amazônia (e.g. Tello e Bayley, 2001). De acordo com estes autores, a intensidade da pesca na região de Iquitos (Peru) provocou redução progressiva de espécies de grande porte e um correspondente incremento de espécies menores, principalmente aquelas do gênero Mylossoma e Triportheus. Espécies menores e numerosas como, por exemplo, curimatídeos e também Triportheus, são usadas em maior escala na pesca comercial quando os peixes maiores e mais apreciados não estão disponíveis (Lowe-McConnell, 1999).

Um outro fator que poderia estar associado ao aumento da produção pesqueira das sardinhas é também a elevação de seu valor no mercado. De acordo com os pescadores, no período de 1990 a 1997, o preço da sardinha era pouco atrativo (aproximadamente R \$ 0,50 por quilo), enquanto que nos últimos anos (1999 a 2004) foram registrados valores variando de $R \$ 1,50$ a $\mathrm{R} \$ 2,50$.

Além disso, o pulso de inundação pode estar também contribuindo com esse aumento, mas resultados mais conclusivos só poderiam ser obtidos mediante o uso de dados de esforço pesqueiro, que não estão disponíveis para a região. Contudo, como visualizado graficamente, os dados sugerem um aumento na produção pesqueira anual das sardinhas precedidos de "cheias mais intensas". Fenômenos como estes já foram registrados para a região do rio Madeira. A "grande cheia" que ocorreu de 1996 para 1997 foi responsável por um considerável incremento na produção pesqueira total calculada no fim do ano de 1997, uma vez que quanto maior o alagamento de grandes áreas de várzea, igapós e lagos centrais, maiores serão os reabastecimentos de indivíduos para a pesca. Segundo pescadores locais isso ocorre quando o nível hidrológico ultrapassa a cota de $1.600 \mathrm{~cm}$. Além disso, cheias mais pronunciadas tendem a permitir maior sucesso reprodutivo, pois o espaço físico favorece menores taxas de competição, predação e maior oferta alimentar (Lowe-McConnell, 1999).

A captura das sardinhas apresentou forte sazonalidade no período analisado, estando intimamente associada à dinâmica de subida e descida dos rios. Essa relação é freqüentemente sugerida por diversos autores em toda a Amazônia para a maioria das espécies de peixes (Boischio, 1992; Batista, 1998; Isaac et al., 2004). A explicação reside, sobretudo, na dispersão dos peixes na extraordinária massa d'água que invade o leito e margens dos rios durante as fases de cheia, diminuindo assim a sua densidade natural e logicamente reduzindo o grau de capturabilidade dos aparelhos de pesca.

As espécies do gênero Triportheus empreendem grandes migrações para fins reprodutivos e esse evento geralmente ocorre entre setembro e outubro no rio Madeira. É fato que os pescadores se aproveitam desta situação para aumentar as capturas destas espécies, bem como de outras, como a jatuarana (Brycon sp.), a curimatã (Prochilodus nigricans) e o jaraqui (Semaprochilodus spp.) (Goulding, 1979). O conhecimento empírico dos pescadores sobre processos ecológicos dos peixes compreende um importante artifício para a efetividade da captura dos estoques, e isso também ajuda a explicar a forte sazonalidade da pesca comercial sobre esse estoque, definindo o período de safra das sardinhas entre setembro e novembro.

A preferência pela utilização dos rios como principal ambiente de pesca possivelmente está associada aos movimentos migratórios das espécies que compõem esta categoria pesqueira. Os aparelhos comumente empregados para a captura de sardinhas 
foram redes de lance e malhadeiras, estando de acordo com os resultados encontrados por Petrere Jr. (1978), onde o autor encontrou além dessas artes de pesca, a utilização ainda da arrastadeira para a pesca no estado do Amazonas.

A tendência à presença de duas modas na estrutura em comprimento poderia estar, primeiramente, demonstrando dois grupos etários recrutados à pesca comercial: o primeiro compreendido aproximadamente até $170 \mathrm{~mm}$, e o segundo de $170 \mathrm{~mm}$ a $380 \mathrm{~mm}$.

Santos (1986/87) verificou a presença de três espécies de sardinhas nos mercados do estado de Rondônia: T. albus, T. angulatus e T. auritus. No entanto, de acordo com Goulding (1979) e com observações pessoais, as maiores participações são de T. angulatus e T. auritus no mercado pesqueiro da cidade de Porto Velho. Diante desta informação, uma hipótese plausível para o padrão bimodal na estrutura em comprimento seria o fato de não ter havido distinção de espécies no momento dos registros da biometria. Desta forma, cada grupo modal da distribuição percentual dos comprimentos totais poderia estar representando uma espécie. As classes entre 60 e $160 \mathrm{~mm}$ poderiam ser representadas em sua maioria por indivíduos de $T$. angulatus, enquanto T. auritus estaria concentrada nas classes maiores. No ano de 2004, no entanto, não foi verificado um padrão bimodal e, além disto, neste ano os indivíduos recrutados foram aparentemente maiores. Estes dados indicam que, em 2004, T. auritus contribui de forma maciça para a produção pesqueira, já que esta espécie, entre as três, é a maior em comprimento (Ferreira et al., 1998).

$\mathrm{Na}$ Amazônia, de todas as espécies de peixes existentes, aproximadamente cem a duzentas são comumente comercializadas. Todavia, estas são tratadas sob a designação de apenas trinta ou cinqüenta nomes ou categorias distintas e com as quais as estatísticas pesqueiras frequentemente trabalham. Desta forma, existem muitos casos em que duas ou mais espécies são agrupadas como apenas uma (Böhlke et al., 1978).

Essa nomenclatura, por um lado, facilita a identificação do peixe pelo pescador e coletor de dados. No entanto, dificulta o acurado entendimento da dinâmica pesqueira, além de refletir nas medidas adotadas para o ordenamento pesqueiro, podendo levar a erros graves (Santos e Santos, 2005). Isso se explica pelo fato de que as espécies biológicas, por mais semelhantes que sejam, podem ocupar nichos especiais e se comportar de maneira distinta na natureza (Lowe-McConnel, 1999).

Queiroz et al. (dados não publicados) verificaram que Triportheus albus e T. angulatus apresentavam padrões diferenciados no uso de determinados trechos do rio Madeira, principalmente para reprodução e alimentação, interferindo em sua distribuição longitudinal. Sugeriram ainda que T. auritus poderia igualmente apresentar diferenças em seus aspectos biológicos quando comparada às outras duas espécies de sardinhas. Estes autores recomendaram que medidas de manejo e conservação das sardinhas do rio Madeira deveriam ser tomadas de forma diferenciada.

Embora os resultados encontrados neste trabalho possam ser utilizados na tomada de decisões referentes ao manejo pesqueiro desta categoria, é importante salientar que são ainda preliminares e não conclusivos. Mesmo que descreva um importante histórico da exploração das sardinhas na região, não esgota a necessidade da manutenção dos estudos sobre a pesca e biologia das espécies que compõem esta categoria de pescado e evidencia a necessidade de separar, no desembarque as espécies facilmente reconhecidas pelos pescadores.

\section{Agradecimentos}

Os autores agradecem à Colônia de Pescadores de Porto Velho Tenente Santana Z1 por disponibilizar os dados usados neste trabalho, aos pescadores pela disposição em contribuir com a pesquisa e a Furnas Centrais Elétricas pelo suporte financeiro na execução do projeto "Diagnóstico da ictiofauna e da pesca comercial na área sob influência dos Aproveitamentos Hidrelétricos Santo Antônio e Jirau", pelo qual os dados de 2003 e 2004 foram obtidos. Ademais, os autores são profundamente agradecidos ao consultor anônimo pelas preciosas sugestões fornecidas. 


\section{Referências}

Amaral, B. D. 2005. Fisheries and fishing effort at the indigenous reserves Ashaninka/Kaxinawá, river Breu, Brazil/Peru. Acta Amazonica, 35 (2): 133-144.

Barthem, R. B. 2004. O desembarque na região de Belém e a pesca na foz amazônica. In: Ruffino, M. L. (ed.). A pesca e os recursos pesqueiros na Amazônia brasileira. Ibama/Pró-Várzea, Manaus, Brasil, p.153-183.

Barthem, R. B.; Fabré, N. N. 2004. Biologia e diversidade dos recursos pesqueiros da Amazônia. In: Ruffino, M. L. (ed.). A pesca e os recursos pesqueiros na Amazônia brasileira. Ibama/PróVárzea, Manaus, Brasil, p.11-55.

Batista, V. S. 1998. Distribuição, dinâmica da frota e dos recursos pesqueiros da Amazônia Central. Tese de Doutorado, Instituto Nacional de Pesquisas da Amazônia/Fundação Universidade Federal do Amazonas, Brasil, 291pp.

Batista, V. S. 2004. A pesca na Amazônia Central. In: Ruffino, M. L. (ed.). A pesca e os recursos pesqueiros na Amazônia brasileira. Ibama/Pró-Várzea, Manaus, Brasil, p.213-244.

Batista, V. S.; Inhamuns, A. J.; Freitas, C. E. C.; Freire-Brasil, D. 1998. Characterization of the fishery in river communities in the low-Solimões/high Amazon region. Fisheries Management and Ecology, 5: 419-435.

Batista, V. S.; Petrere Jr., M. 2003. Characterization of the commercial fish production landed at Manaus, Amazonas state, Brazil. Acta Amazonica, 33 (1): 53-66

Böhlke, J. E.; Weitzman, S. H.; Menezes, N. A. 1978. Estado atual da sistemática dos peixes de água doce da América do Sul. Acta Amazonica, 8 (4): 657-677.

Boischio, A. A. P. 1992. Produção pesqueira em Porto Velho, Rondônia (1984-89) - alguns aspectos ecológicos das espécies comercialmente relevantes. Acta Amazonica, 22 (1): 163-172.

Cerdeira, R. G. P.; Ruffino, M. L.; Isaac, V. J. 1997. Consumo de pescado e outros alimentos pela população ribeirinha do lago Grande de Monte Alegre, PA - Brasil. Acta Amazonica, 27 (3): 213 228.

Ferreira, E. J. G.; Zuanon, J. A. S.; Santos, G. M. 1998. Peixes comerciais do Médio Amazonas: região de Santarém, Pará. Edições IBAMA, Brasília, Brasil, 214pp.
Goulding, M. 1979. Ecologia da pesca do rio Madeira. INPA, Manaus, Brasil, 154pp.

Isaac, V. J.; Silva, C. O.; Ruffino, M. L. 2004. A pesca no Baixo Amazonas. In: Ruffino, M. L. (ed.). A pesca e os recursos pesqueiros na Amazônia brasileira. Ibama/PróVárzea, Manaus, Brasil, p.185-211.

LEME, Engenharia S.A. 2005. Estudo de Impacto Ambiental dos Aproveitamentos Hidrelétricos Santo Antônio e Jirau, Rio Madeira - RO. Capítulo IV - Área de Influência Direta dos Aproveitamentos Hidrelétricos de Jirau e Santo Antônio, Tomo B, Volume 5/8, Diagnóstico Ambiental da Área de Influência Direta, Meio Biótico, Ictiofauna e Recursos Pesqueiros. Relatório 6315 - RT - G90 - 001. Maio/2005. p IV-755 - IV-916

Lowe-McConnell, R. H. 1999. Estudos ecológicos de comunidades de peixes tropicais. Edusp, São Paulo, Brasil, 534pp.

Petrere Jr., M. 1978. Pesca e esforço de pesca no Estado do Amazonas. II. Locais de pesca, aparelhos de captura e estatísticas de desembarque. Acta Amazonica, 8 (Supl. 2): 1-54.

Petrere Jr., M. 1982. Manejo de estoques pesqueiros na Amazônia. Informe Científico. UNESP, Rio Claro, Brasil, 12pp.

Santos, G. M. 1986/87. Composição do pescado e situação da pesca no estado de Rondônia. Acta Amazonica, 16/17: 43-84.

Santos, G. M.; Ferreira, E. J. G. 1999. Peixes da bacia Amazônica. In: Lowe-McConnell, R. H. (ed.). Estudos ecológicos de comunidades de peixes tropicais. Edusp, São Paulo, Brasil, p.345-354.

Santos, G. M.; Santos, A. C. M. 2005. Sustentabilidade da pesca na Amazônia. Acta Amazonica, 19 (54): 165-182.

Tello, S.; Bayley, P. 2001. La pesquería comercial de Loreto con énfasis en el análisis de la relación entre captura y esfuerzo pesquero de la flota comercial de Iquitos, cuenca del Amazonas (Perú). Folia Amazónica, 12 (1-2): 123-139.

Viana, J. P. 2004. A pesca no Médio Solimões. In.: Ruffino, M. L. (ed.). A pesca e os recursos pesqueiros na Amazônia brasileira. Ibama/PróVárzea, Manaus, Brasil, p.245-268.

Zar, J. H. 1999. Biostatistical Analysis. Prentice Hall, Englewood Cliffs, New Jersey, USA, 471pp. 